\title{
Reusing Data During Speech Pauses in an NLMS-based Acoustic Echo Canceller
}

\author{
Fredric Lindstrom* ${ }^{*}$, Christian Schüldt ${ }^{\dagger}$, Ingvar Claesson $^{\dagger}$ \\ ${ }^{*}$ Konftel AB, Research and Development, Box 268, SE-90106, Umeå, Sweden, fli@ieee.org \\ ${ }^{\dagger}$ Blekinge Institute of Technology, Department of Signal Processing, SE-37225, Ronneby, Sweden
}

\begin{abstract}
Fast convergence of the adaptive filter in an acoustic echo cancellation based hands-free communication system is desirable as it implies more periods of possible full-duplex communication. This paper presents a normalized least mean square (NLMS)-based algorithm, targeted for acoustic echo cancellation based units equipped with large external memory. The proposed algorithm utilizes unused processing resources in periods of silence, thus no extra complexity as compared with the conventional NLMS algorithm is required. The improvements obtained by the proposed algorithm are verified through simulated, as well as through real acoustic systems.
\end{abstract}

\section{INTRODUCTION}

Modern high quality teleconferencing equipment use acoustic echo cancellation (AEC) [1] in order to provide full-duplex communication. An AEC processing unit generally consists of an adaptive filter process in which the adaptive filter tries to estimate the impulse response of the loudspeaker-enclosuremicrophone (LEM) system. Several algorithms have been proposed for the adaptation of the filter, e.g. the least mean square (LMS), normalized LMS (NLMS), affine projection and recursive least squares algorithms [2]. The NLMS is perhaps the most widely used algorithm thanks to its low-complexity and robustness.

In a normal two-way telephone speech conversation a talker is active for only about $35 \%$ of the time [3]. Thus, the loudspeaker is silent for an average of $65 \%$ of the time. When the loudspeaker is silent there is no signal driving the adaptive filter in the AEC and the computational resources allocated for the adaptation is not used. This paper proposes an algorithm which uses these computational resources to improve the convergence of the adaptive filter. Several data reusing methods have been proposed earlier, an overview is provided in [4]. This paper differs in that it utilizes silent periods to increase convergence without increasing complexity, and in that it uses old data in a time span of several seconds. Since internal (i.e. on-chip) memory is generally a costly and limited resource, the proposed method is intended for systems equipped with relatively cheap external (i.e. off-chip) memory, e.g. units containing a SDRAM, a compact flash or a hard disk. Copying data to and from the external memory is typically performed though direct memory access (DMA), used in parallel with the calculations and thus requiring no significant additional complexity. The application presented in this paper is for acoustic echo cancellation, but the general idea can be applied to line echo cancellation as well.

\section{The CONVEnTIONAL NLMS}

The conventional NLMS algorithm is defined by [2],

$$
\begin{aligned}
& \hat{y}(k)=\mathbf{x}(k)^{T} \hat{\mathbf{h}}(k) \\
& e(k)=y(k)-\hat{y}(k) \\
& \hat{\mathbf{h}}(k+1)=\hat{\mathbf{h}}(k)+\mu \frac{e(k) \mathbf{x}(k)}{\|\mathbf{x}(k)\|^{2}+\epsilon}
\end{aligned}
$$

where $x(k)$ is the loudspeaker signal, $y(k)$ the microphone signal, $\hat{\mathbf{h}}(k)=\left[\hat{h}_{0}(k), \cdots, \hat{h}_{N-1}(k)\right]$ the $N$-length finite impulse response (FIR) adaptive filter used to model the LEM, $\mathbf{x}(k)=[x(k), \cdots, x(x-N+1)]$ the regressor vector, $e(k)$ the error or residual echo signal, $\mu$ is the step-size control parameter, and $\epsilon$ is the regularization parameter.

\section{THE PROPOSED ALGORITHM}

The basic idea in this paper is to use available external memory to store large parts of data and then reuse this data in the adaptive process when no current filter convergence data is available, i.e. during periods when the loudspeaker is silent.

\section{A. Storing old data}

Old samples of the loudspeakers signal $x(k)$ and the microphone signal $y(k)$ are saved in two arrays, $\mathbf{x}_{o}=$ $\left[x_{o}(0), \cdots, x_{o}(I-1)\right]$ and $\mathbf{y}_{o}=\left[y_{o}(0), \cdots, y_{o}(I-1)\right]$, both of length $I$. Since new data is saved continuously during operation, data in the arrays will be overwritten. For efficient run-time operation, the two arrays should be implemented as circular buffers. The large size arrays $\mathbf{x}_{o}$ and $\mathbf{y}_{o}$ are allocated in external memory. All other arrays and parameters in this paper are assumed to be allocated in internal memory.

Storing data should only be performed in periods when $x(k)$ is active. Activity of $x(k)$ can be evaluated by a simple activity detector based on an averaging of the squared signal

$$
\bar{x}(k)=\left(1-\gamma_{x}\right) \bar{x}(k-1)+\gamma_{x} x^{2}(k),
$$

where $\gamma_{x}$ is an averaging constant, and $x(k)$ is considered active whenever $\bar{x}(k)>T_{x}$, where $T_{x}$ is a constant threshold.

Since speech has a bursty character and $I$ typically is set large, the array $\mathbf{x}_{o}$ will contain several different segments of continuous speech data. In this algorithm, a maximum of $J$ such data segments are allowed. These segments of continuous speech data are denoted $D_{j}$ where $j=0, \cdots, J-1$. The index of the first element in each data segment is denoted $d_{j}$, i.e. $x_{o}\left(d_{j}\right)$ is the first element in $D_{j}$. These $J$ indexes $d_{j}$ are 
ordered in an array $\mathbf{d}=\left[d_{0}, \cdots, d_{J-1}\right]$, see figure 1 . This indexing is used in order to handle the fact that the length of each data segment is not known in advance.

During speech activity, samples are written into the external memory. The data segment which samples currently are written to is denoted $D_{w}$. During speech pauses, samples are read from external memory. The segment which samples currently are read from is denoted $D_{r}$. Since $\mathbf{x}_{o}$ is circular, the data segments will eventually be overwritten. The oldest data segment not yet overwritten is denoted $D_{s}$ and is the segment which the reading will start from after a reading wrap-around in the circular array $\mathbf{x}_{o}$. To keep track of these three segments, three integer indexes are required $w, r, s$ with $0 \leq w, s, r \leq J-1$. Thus $x_{o}\left(d_{w}\right), x_{o}\left(d_{r}\right), x_{o}\left(d_{s}\right)$, are the first elements in $D_{w}, D_{r}, D_{s}$, respectively.

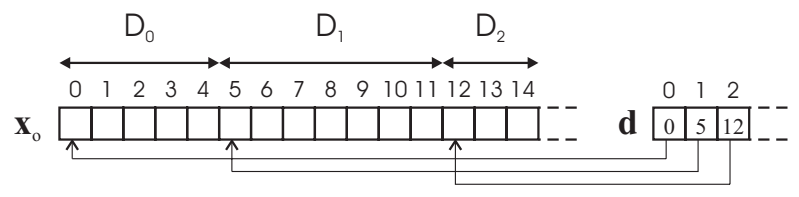

Fig. 1. Schematic picture of $\mathbf{x}_{O}$ and $\mathbf{d}$

The definitions presented above are used in the proposed storing procedure, given by listing 1 , which checks if $x(k)$ is active (LINE 1), and if so, the data is stored (LINE 2 and LINE 3) and $m$, the number of stored samples in the current write segment, is updated (LINE 4).

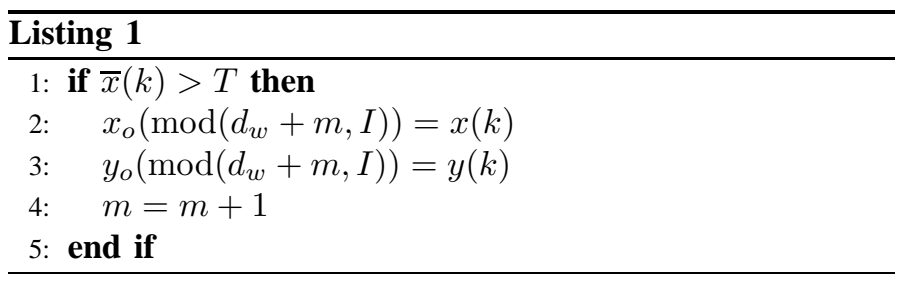

Note that wrap-around is performed by the modulo operation $\bmod (\cdot, \cdot)$ and is done to ensure that stored data and used indexes are contained within the allocated arrays.

The estimate of the echo, see equation (1), is calculated using an $N$ length regressor vector. To be consistent with the FIR modeling of the unknown LEM system, the samples in the regressor vector should be from a continuous set of data. If the length of a speech segment in the array $\mathbf{x}_{o}$ is less than $N$, it should thus not be used since it will not be able to fill the regressor vector. Further, a data segment being only a few samples longer than $N$ can only be used for a few samples before switching to the next data segment. Thus, the length of a data segment should be greater than a minimum length $L_{\mathrm{MIN}}$, with $L_{\mathrm{MIN}} \geq N$. Note that $L_{\mathrm{MIN}}$ defines $J$ (the maximal number of segments in the fixed length vector $\mathbf{x}_{o}$ ) through $J \geq$ $I / L_{\mathrm{MIN}}$.

The minimal length requirement of the data segments is assured through listing 2, where a check if a segment of continuous speech has just ended is performed (LINE 1). Then

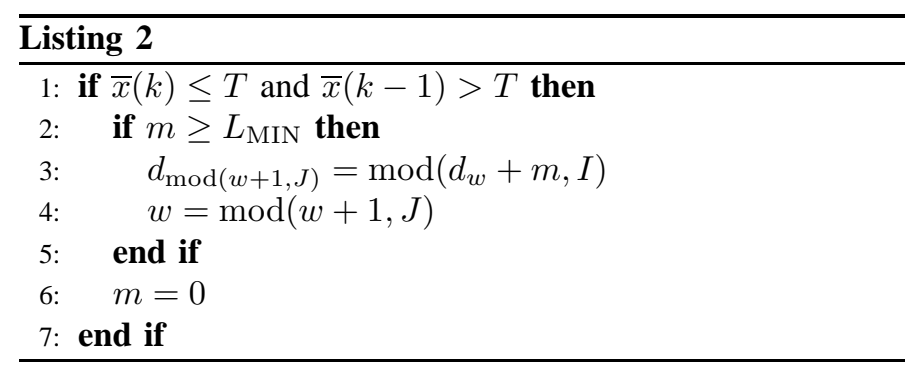

there is a check if the data segment is of sufficient length (LINE 2). If so, (LINE 3-4) finalizes the storing of the data segment, by first updating the location of the first element in the current write segment (LINE 3) and then the index of current write segment $w$ (LINE 4). Thereafter, $m$, i.e. the number of stored samples, is reset (LINE 6). If the segment is not of sufficient length the current write index is not updated, only $m$ is reset (LINE 6), i.e. the segment is not stored.

Since the LEM system is non-stationary, a time stamp should be assigned to each data segment to ensure that not too old data is used. If such an approach is to be reliable, the data segments cannot be too long. Assuring a maximum length $L_{\mathrm{MAX}}$ of the

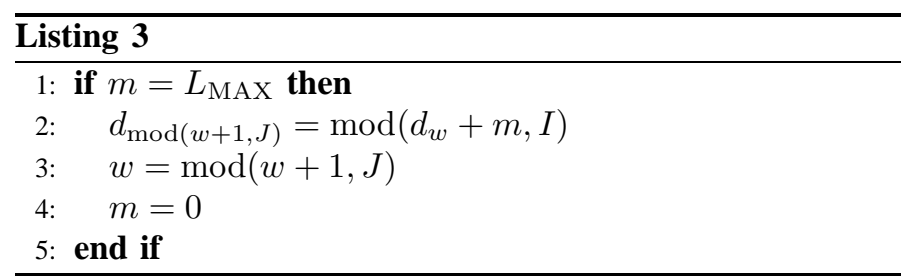

data segments can be obtained through listing 3, which starts with a check if a data segment have reached its maximum allowed length (LINE 1). If so, the storing of the segment is finalized (LINE 2-3), and $m$ is reset (LINE 4).

If the start segment $D_{s}$ is overwritten, the start index $s$ needs to be updated, as shown in listing 4, where $s$ is updated (LINE 2) if the first element in the start segment is overwritten (LINE 1).

\begin{tabular}{l}
\hline Listing $\mathbf{4}$ \\
\hline $1:$ if $d_{w}+m=d_{s}$ then \\
2: $\quad s=\bmod (s+1, J)$ \\
3: end if \\
\hline
\end{tabular}

\section{B. Adaptive filtering}

In both the proposed and the conventional NLMS, the filter update is performed in the same manner when the speech signal $x(k)$ is active. Both solutions update the filter with the same update vector

$$
\Delta(k)=\mu \frac{e(k) \mathbf{x}(k)}{\|\mathbf{x}(k)\|^{2}+\epsilon} .
$$

If $x(k)$ is not active, i.e. silent, the conventional NLMS omits the updating, while the proposed algorithm uses old data to 
obtain a filter update vector $\Delta_{o}(k, i)$,

$$
\Delta_{o}(k, i)=\mu \frac{e_{o}(k) \mathbf{x}_{o_{N}}(i)}{\left\|\mathbf{x}_{o_{N}}(i)\right\|^{2}+\epsilon},
$$

where $i$ is an index $0 \leq i \leq I-1$ denoting which of the stored samples to use in the update, and $\mathbf{x}_{o_{N}}(i)=\left[x_{o}(i), \cdots x_{o}(i-\right.$ $N+1)$ ] is the corresponding regressor vector. If $i-N+1$ is less than zero, appropriate wrap-around is performed. The proposed updating scheme is thus as given in listing 5, where (LINE 1) checks for activity and if activity is detected, the filter update is performed according to the conventional NLMS (LINE 2-4). If $x(k)$ is not active, the filter $\hat{\mathbf{h}}(k)$ is updated using previously stored data (LINE 6-8). Finally, the index $i$ is updated (LINE 9).

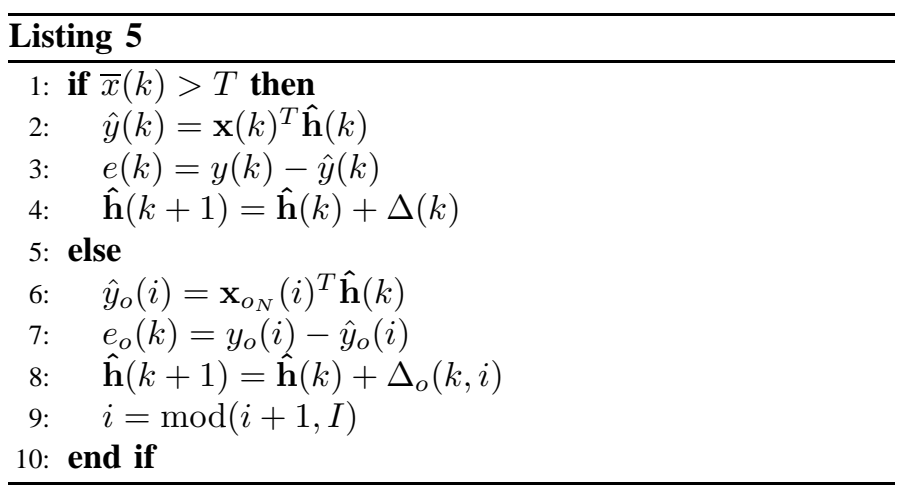

If $i$ reaches the current write segment, i.e. if $i=d_{w}$, a wrap-around is needed. Further, if the index $i$ reaches the next data segment to be read, i.e. if $i=d_{r+1}$, an update of the whole regressor vector $\mathbf{x}_{O_{N}}(i)$ is needed, since the regressor vector should only contain data from a single segment. Thus, the operations are as given in listing 6, where if the index $i$ has reached the current write data segment $D_{w}$ (LINE 1), the index needs to be wrapped around to the oldest available data segment $D_{s}$. The corresponding modification of index $i$ (LINE 2) and the current read segment index $r$ is set to the start segment index $s$ (LINE 3). If the index $i$ has reached the next data segment to be read $D_{r+1}$ (LINE 4), the regressor vector $\mathbf{x}_{o}$ needs to be completely updated with samples from the new data segment. Thus, $i$ is then increased with $N$ (LINE 5), which implies that the regressor vector is filled with values from the new segment, and the current read data segment index $r$ is updated (LINE 6).

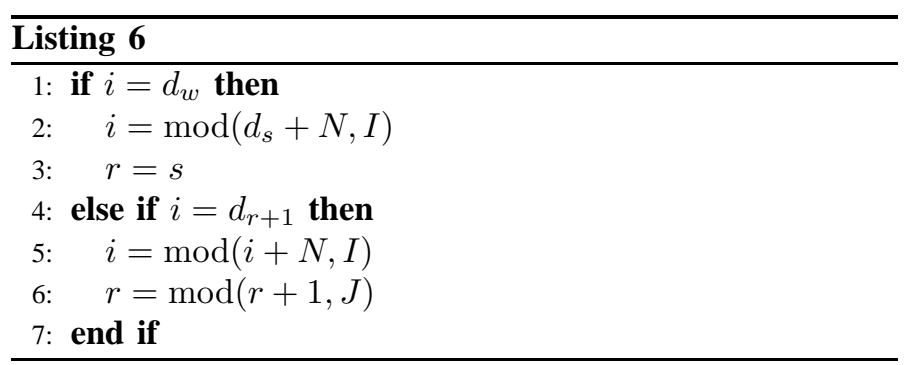

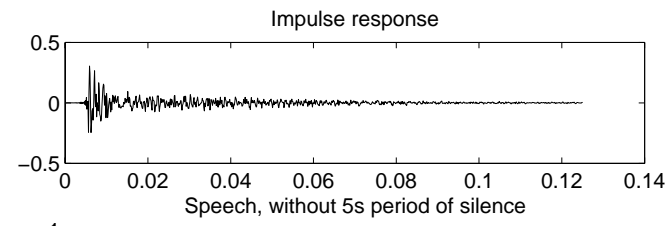
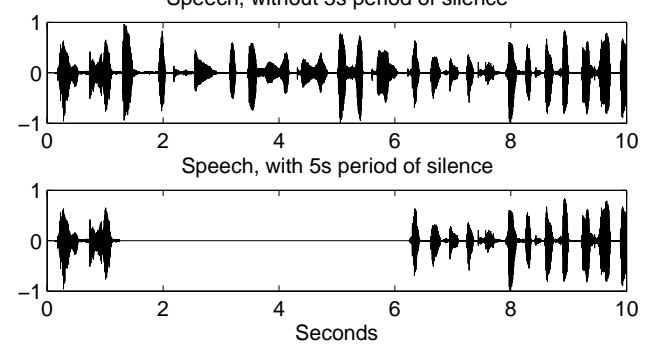

Fig. 2. Impulse response and signals
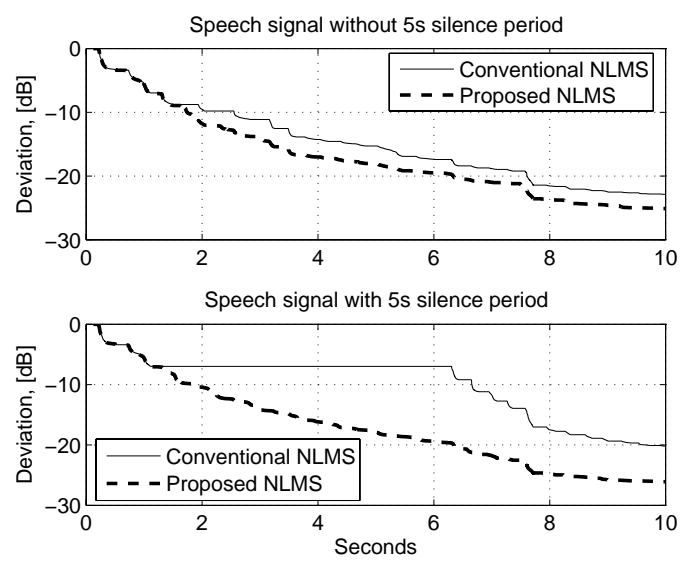

Fig. 3. Simulated acoustic system

\section{Simulated SYSTEM}

In the simulated system, the microphone signal was obtained by filtering the loudspeaker signal with a FIR model of a real LEM system $\mathbf{h}$, see figure 2 . Two different loudspeaker signals were used, where one contained a $5 \mathrm{~s}$ period of silence, see figure 2. The parameter settings were as given in table I and the sampling frequency was $8 \mathrm{kHz}$. Evaluation of the proposed system was performed in parallel with the conventional NLMS. The normalized squared system deviation [2]

$$
M(k)=\frac{\|\mathbf{h}-\hat{\mathbf{h}}(k)\|^{2}}{\|\mathbf{h}\|^{2}}
$$

was used for evaluation.

In figure 3 it can be seen that for the speech signal without a long period of silence, the proposed method performs slightly better than the conventional NLMS, while for the speech signal with a $5 \mathrm{~s}$ period of silence, the proposed method significantly improves convergence.

\section{REAL SYSTEM}

In the real system evaluation, the microphone signal was obtained by feeding the speech signals in figure 2 to a real loudspeaker and recording a real microphone signal. The setup was done in a small office with a distance of approximately 


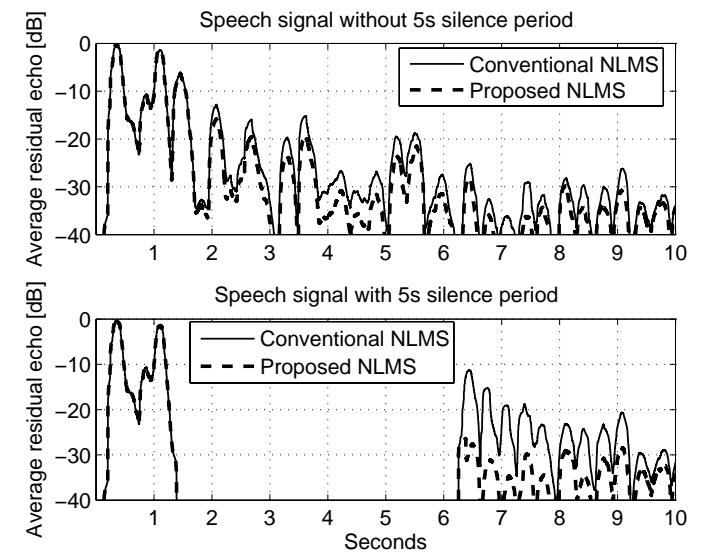

Fig. 4. Real acoustic system

\begin{tabular}{|ll|ll|ll|}
\hline$I$ & 32000 & $J$ & 22 & $N$ & 1000 \\
$\gamma_{x}$ & 0.99 & $T_{x}$ & 0.03 & $\mu$ & 0.5 \\
$L_{\mathrm{MIN}}$ & 1500 & $L_{\mathrm{MAX}}$ & 8000 & $\epsilon$ & 4 \\
\hline
\end{tabular}

TABLE I

PARAMETER SETTINGS

$40 \mathrm{~cm}$ between the loudspeaker and the microphone. For a real system, the deviation measure cannot be used, since the true impulse response is unknown. In this case, the average power of the two algorithms echo residual signals $e(k)$ are evaluated instead. In figure 4 it can be seen that for the signal without a long silent period the proposed method yields slightly better echo cancellation. For the signal with a long silence period, the echo of the the conventional NLMS is around $-10 \mathrm{~dB}$ after 6.5 seconds, while it for the proposed method is reduced to below $-25 \mathrm{~dB}$, i.e. a significant improvement. Thus, the proposed solution can provide a significant convergence speed increase for an AEC application.

\section{CONCLUSIONS}

In this paper an algorithm which improves the convergence of the NLMS algorithm in an acoustic echo cancellation unit equipped with a large external memory was presented. The algorithm exploits the signal processing resources that are not efficiently used during periods of silence in the conventional NLMS. Thereby the proposed algorithm does not imply any significant increase in complexity compared to the conventional NLMS. The possible improvements obtained through the use of the proposed algorithm were shown through evaluation of a simulated as well as a real acoustic environment.

\section{ACKNOWLEDGMENT}

Thanks to the Swedish Knowledge Foundation (KKS) for funding.

\section{REFERENCES}

[1] E. Hänsler and G. Schmidt, Acoustic Echo and Noise Control: A Practical Approach. Wiley, 2004.

[2] S. Haykin, Adaptive Filter Theory, 4th ed. Prentice-Hall, 2002.

[3] P. T. Brady, "A technique for investigating on-off patterns of speech," Bell. Syst. Tech. J., vol. 44, pp. 1-22, 1965.
[4] R. A. Soni, K. A. Gallivan, and W. K. Jenkins, "Low-complexity data reusing methods in adaptive filtering," IEEE Transactions on Signal Processing, vol. 52, no. 2, pp. 394-405, 2004. 